\title{
The Dorsolateral Prefrontal Cortex Contributes to Successful Relational Memory Encoding
}

\author{
Linda J. Murray and Charan Ranganath \\ Center for Neuroscience and Department of Psychology, University of California at Davis, Davis, California 95616
}

\begin{abstract}
Results from neuroimaging studies of long-term memory (LTM) encoding have contributed to the view that the ventrolateral prefrontal cortex (VLPFC) contributes to successful LTM formation, whereas the dorsolateral prefrontal cortex (DLPFC) does not. We hypothesized that the DLPFC does contribute to LTM, but under specific circumstances. That is, the DLPFC may be critical for building relationships between items during on-line processing, and this may promote LTM for associations between items. We used event-related functional magnetic resonance imaging (fMRI) to test this hypothesis by examining brain activity during sequential encoding of unrelated word pairs. During presentation of the second ("target") word in each pair, subjects either made a semantic judgment specific to the target word ("item-specific" trials), or a semantic judgment that involved a comparison between the target word and the first word in the pair ("relational" trials). Behaviorally, recognition memory for target words was equivalent between the two trial types but associative recognition of studied word pairs was significantly greater for relational trials. fMRI results showed that DLPFC activity was greater during relational compared with item-specific encoding and that DLPFC activity predicted successful memory for associations but not successful item memory. Activity in the VLPFC was also greater for relational compared with item-specific encoding, but VLPFC activation predicted successful memory for both associations and items. These results support the view that the DLPFC may contribute to LTM through its role in active processing of relationships during encoding, whereas the VLPFC may have a more general role in promoting successful LTM formation.
\end{abstract}

Key words: prefrontal; encoding; memory; relational; dorsolateral; fMRI

\section{Introduction}

Evidence from neuropsychological studies is consistent with the idea that regions in the lateral prefrontal cortex (PFC) implement cognitive control processes that contribute to successful episodic long-term memory (LTM) encoding (Ranganath and Blumenfeld, 2007). Furthermore, neuroimaging studies have shown that activation in the ventrolateral PFC (VLPFC), at or near Brodmann's areas (BA) 44, 45, and 47, is consistently increased during encoding of items that are subsequently remembered, compared with items that are later forgotten (Paller and Wagner, 2002; Ranganath and Blumenfeld, 2007). Interestingly, few imaging studies have linked activity in the dorsolateral PFC (DLPFC; BA 9 and 46) with successful LTM formation, and some have even found that DLPFC activation is increased for items that are later forgotten (Otten and Rugg, 2001; Wagner and Davachi, 2001; Daselaar et al., 2004). These results have been interpreted to suggest that the DLPFC implements processes that do not contribute to successful LTM formation, such as response selection or task switching (Otten et al., 2002).

Received Dec. 7, 2006; revised March 21, 2007; accepted March 26, 2007.

This work was supported by National Institutes of Health Grants R01 MH068721 and P01NS40813. We thank Aaron Heller, Rob Blumenfeld, Rachel Diana, Andrew Yonelinas, Neal Kroll, Colleen Parks, Craig Brozinsky, Deborah Hannula, and Andrew Haskins for insightful comments.

Correspondence should be addressed to Linda J. Murray, Center for Neuroscience, University of California at Davis, 1544 Newton Court, Davis, CA 95616. E-mail: Imurray@ucdavis.edu

D0I:10.1523/JNEUROSCI.0406-07.2007

Copyright $\odot 2007$ Society for Neuroscience $\quad$ 0270-6474/07/275515-08\$15.00/0
In contrast to this idea, some recent results suggest that the DLPFC contributes to LTM formation under specific circumstances. For example, Blumenfeld and Ranganath (2006) examined DLPFC activation during two working memory tasks; one involved processing relationships between sets of three words and the other emphasized rehearsal of the words. Only the task that emphasized relationships showed activation that was correlated with subsequent LTM for the memory sets. In a different study, Staresina and Davachi (2006) reported that DLPFC activation during word encoding was correlated with subsequent LTM performance on a free recall test but not with performance on tests of item recognition memory. Critically, encoding of relationships between items disproportionately benefits performance on free recall tests, compared with recognition (Tulving, 1962). Thus, it is possible that DLPFC activation in these studies was specifically correlated with successful LTM under conditions in which performance on the memory test benefited from the processing of relationships among items at encoding (Ranganath and Blumenfeld, 2007).

Here, we tested the hypothesis that the DLPFC is critical for building relationships between items during on-line processing, which in turn may facilitate LTM by strengthening inter-item associations (Bower, 1970). We used event-related functional magnetic resonance imaging (fMRI) to examine prefrontal activation during sequential encoding of word pairs. During presentation of the second ("target") word in each pair, subjects performed an encoding task that encouraged them to build 


\section{Experimental Design}

Item-specific trials:

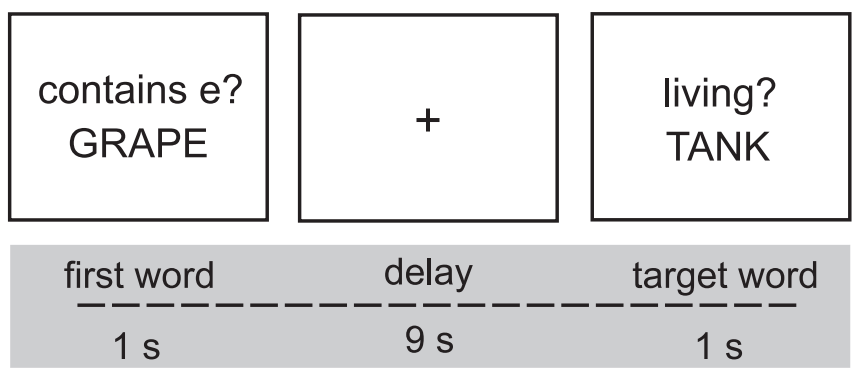

Relational trials:

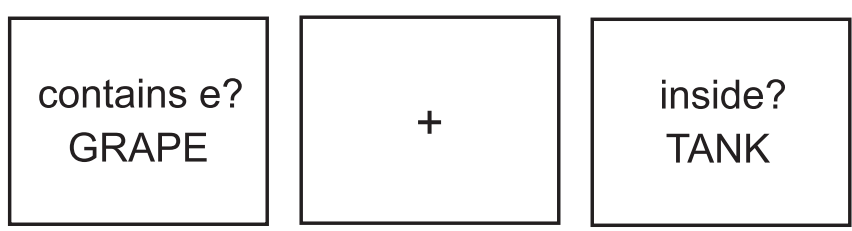

Figure 1. Example stimuli and task timing for encoding phase.

relationships between the two words in each pair ("relational" trials) or to focus on specific semantic attributes of the target word ("item-specific" trials) (see Fig. 1). After scanning, participants were tested on memory for the individual words presented during the study phase and for the associations between the word pairs. We hypothesized that DLPFC activation should be (1) greater during relational trials than during item-specific trials and (2) predictive of subsequent associative memory. For comparison purposes, we also investigated activation in the VLPFC, parahippocampal cortex (PHC), and fusiform gyrus; regions that have consistently been implicated in successful memory encoding (Paller and Wagner, 2002).

\section{Materials and Methods}

Participants. Seventeen (eight male; aged 18-35) participants were recruited from the University of California at Davis (UC Davis) student community. Participants gave informed consent before the experiment and were paid for their participation. Participants were excluded from analyses if the mean response time in any trial condition was $>3000 \mathrm{~ms}$. This resulted in the exclusion of two participants. Additionally, one participant was excluded because behavioral data from the scanner was not recorded (because of equipment malfunction).

Materials. Stimuli in this experiment consisted of 384 words selected from the Medical Research Council Psycholinguistic Database (http:// www.psych.rl.ac.uk/MRC_Psych_Db.html). The words were three to nine letters in length (mean, 5.3; SD, 1.5) with a mean Kucera-Francis written frequency of 22 . These words were used to construct six separate lists of 64 words matched for length and frequency. Four of the word lists were used for the study task. Each word list was used to generate either the first word or the target word in each word pair for either item-specific or relational trials (see below for descriptions of terms). This assignment was counterbalanced across subjects. Words from the remaining two lists were used as foils for the subsequent LTM item recognition test.

Behavioral procedure. During the scanning phase, participants first performed a visuomotor response task, the results of which were used to empirically estimate a subject-specific hemodynamic response function (HRF; see below). Next, participants were scanned during incidental encoding of word pairs (Fig. 1). On each trial, a word was shown for $1 \mathrm{~s}$ (the first word), along with the instruction "contains e?". Participants made a button response to indicate whether the word contained the letter "e". After a $9 \mathrm{~s}$ delay, a second word appeared for $1 \mathrm{~s}$ (the target word), along with an instruction indicating the type of decision that was to be made. During the delay period, participants were instructed to subvocally rehearse the first word until the target word was shown. Because the sequence of trials was random, participants could not predict which task was to be performed with the target word. On item-specific trials, the instruction "living?" was presented above the target word, prompting participants to make a button response to indicate whether the word represented something living or nonliving. On relational trials, the instruction "inside?" was written above the target word, prompting participants to indicate whether the item represented by the target word could fit inside the item represented by the first word, or vice versa. For example, if the first word was "grape" and the second word was "tank," the participant had to decide whether a grape could fit inside a tank or whether a tank could fit inside a grape. For all tasks, participants used a response box to indicate "yes" (index finger) and "no" (middle finger) with their right hand. Participants performed a total of 64 item-specific and 64 relational trials, which were presented in a pseudorandom sequence.

After the scanning session, participants completed two surprise memory tests. First, they completed an item recognition test in which all 256 words (first words and target words) from the study phase were shown individually on a computer screen, randomly intermixed with 128 novel foils. Participants were instructed to respond "remember" if they could recollect specific details regarding the occurrence of a word during the study phase, to respond "familiar" if they were confident that they had studied the word but did not recollect any details of the study event, and to respond "new" if they did not think the word was studied during the scan (Tulving, 1985). Results from this test were used to estimate the degree to which recognition was supported by recollection and familiarity. These estimates were computed under the assumption that recollection and familiarity each independently contribute to recognition judgments (Yonelinas and Jacoby, 1995), using the following equations, where $p$ is probability, $R$ is remember response, and $F$ is familiar response: $p($ recollection $)=p(R \mid$ studied $)-p(R \mid$ unstudied $)$ and $p($ familiarity $)=p(K \mid$ studied $) /(1-p(R \mid$ studied $)-p(K \mid$ unstudied $) /(1-$ $p(R \mid$ unstudied $)$. Estimates were calculated separately for first words and target words in each condition.

Next, participants were tested on memory for the associations between the words studied in the scanner. On each trial, a target word from a studied word pair was first presented on a computer screen for $1 \mathrm{~s}$ and then replaced by two words presented side-by-side: the word that was paired with the target word in the study phase and a foil word that had been paired with a different target word. The left-right placement of these two words (correct match and incorrect match) was randomized across trials. Subjects were instructed to select the word that they thought was paired with the target word and rate whether they were "confident" or "less sure" of their decision (we will refer to these responses as highconfidence and low-confidence responses, respectively). Subjects were instructed to give a high-confidence response only if they could remember the specific associate of the target word. If they were not able to remember the associate, but did remember that the foil word was paired with a different word in the scan session, they were instructed to make a low-confidence response.

MRI acquisition and processing. MRI data were collected on a 3T Siemens AG (Erlangen, Germany) Trio scanner at the UC Davis Imaging Research Center. Functional images sensitive to blood oxygenation leveldependent (BOLD) contrast were acquired using a gradient echo-planar imaging (EPI) sequence with the following parameters: repetition time (TR), $2000 \mathrm{~ms}$; echo time (TE), $25 \mathrm{~ms}$; flip angle, $90^{\circ}$; field of view (FOV), $220 \mathrm{~mm}$; matrix size, $64 \times 64$. Each volume consisted of 34 contiguous 3.4-mm-thick axial slices, resulting in a voxel size of $3.4375 \times 3.4375 \times$ $3.4 \mathrm{~mm}$. Coplanar T2-weighted images were acquired using a spin-echo sequence (TR, $4000 \mathrm{~ms}$; TE, $109 \mathrm{~ms}$; flip angle $90^{\circ}$; matrix size, $512 \times 512$; slice thickness, $3.4 \mathrm{~mm}$ ) and high-resolution, T1-weighted images were also acquired using an MP-RAGE (magnetization-prepared rapidacquisition gradient-echo) sequence (TR, $1750 \mathrm{~ms}$; TE, $2.93 \mathrm{~ms}$; flip angle, $12^{\circ}$; matrix size, $256 \times 256$ ).

fMRI data preprocessing was performed with Statistical Parametric 
Mapping (SPM2; Wellcome Department of Cognitive Neurology, London, UK) software for all subjects. EPI images were sinc interpolated in time to correct for between-slice timing differences in image acquisition, realigned using a six-parameter, rigid-body transformation algorithm, spatially normalized to the template from the International Consortium for Brain Mapping Project (Cocosco et al., 1997), resliced into $3.5 \mathrm{~mm}$ isotropic voxels, and spatially smoothed with an $8 \mathrm{~mm}$ full-width at half-maximum Gaussian filter.

MRI data analysis. Activity changes during each phase of each trial were deconvolved using a modified general linear model (Worsley and Friston, 1995) as implemented in the VoxBo software package (available at www.voxbo.org). The basic assumption behind this approach is that the time course of BOLD signal changes on each trial reflects a linear combination of temporally distinct neural activity patterns that are each convolved with the HRF (Boynton et al., 1996). Covariates modeling BOLD signal changes during the first word, delay period, and target word on each trial were constructed by convolving vectors of expected neural activity associated with each of these components with a subject-specific HRF estimated from responses in the central sulcus during the visuomotor response task (Handwerker et al., 2004). Data from the visuomotor response task were not available for three subjects, and for these subjects, covariates were constructed by convolving the vector of expected neural activity with the "canonical" HRF included in SPM2. The onset and offset of delay period vectors were spaced apart from the first word and target word vectors to minimize the possibility that the delay covariate would model activity attributable to the first word and target word periods (for relative timing of covariates as well as information on collinearity estimates, see supplemental material, available at www.jneurosci.org).

BOLD responses were modeled separately for each phase of itemspecific and relational trials. Additional analyses modeled the trial phases as a function of subsequent performance on the item and associative memory tests (for more information on the number of trials in each subsequent memory bin, see supplemental material, available at www. jneurosci.org) For analyses of subsequent memory effects on the item memory test, one covariate modeled activation to target words that were subsequently given a remember response and another covariate modeled activation to target words that were subsequently given either a familiar or new response. Data from one participant was excluded from the item subsequent memory analysis because of a technical problem in collecting responses. For analyses of subsequent memory effects on the associative memory test, one covariate modeled components of trials on which the association was successfully remembered with high confidence and another modeled components of trials on which either the association was recognized with low confidence or an incorrect response was made. One participant was excluded from this analysis because of an inadequate number of correct high-confidence responses.

In addition to the covariates of interest in the analyses described above, separate nuisance covariates were included to model trial phases for which no button press was made in response to the relevant encoding task. Additional covariates modeled global signal changes that could not be accounted for by variables in the design matrix (Desjardins et al., 2001), baseline shifts across scanning runs, and an intercept.

After single-subject analyses, a contrast image was calculated for each participant by calculating the difference in parameter estimates between covariates modeling target word activation during relational and itemspecific trials. These images were entered into a second-level, one-sample $t$ test, in which the mean value across participants at each voxel was tested against zero. The resulting $t$ maps were thresholded using a procedure to constrain the map-wise false discovery rate to $p<0.05$ (Genovese et al., 2002). For visualization purposes, thresholded statistical parametric maps were overlaid on T1-weighted images using MRIcro software (Rorden and Brett, 2000). Additional analyses were performed on regions of interest (ROIs), which were functionally defined from the contrast of target word activation between relational and item-specific trials. The borders of the ROIs were delineated by the left middle frontal (DLPFC), inferior frontal (VLPFC), parahippocampal (PHC), and fusiform gyri.
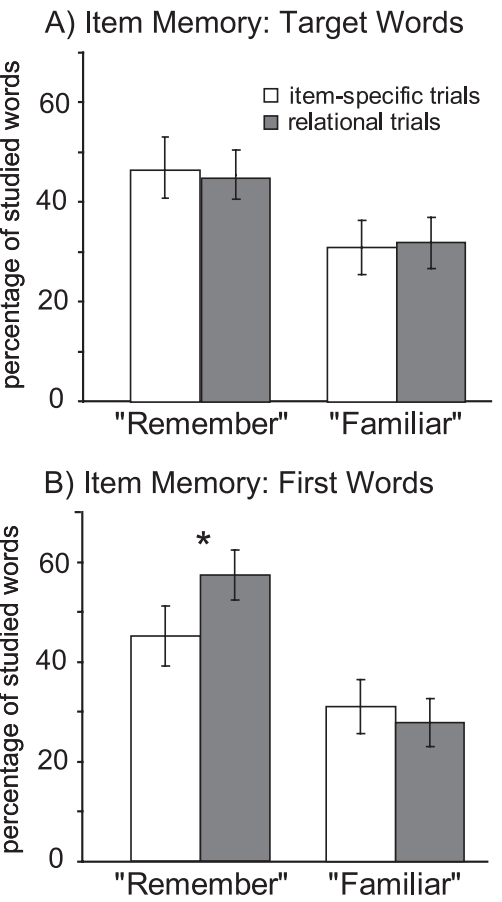

Figure 2. $\quad \boldsymbol{A}$, Mean percentage of remember and familiar responses to target words for item-specific (open bars) and relational (shaded bars) trials. $\boldsymbol{B}$, Mean percentage of remember and familiar responses to first words for item-specific and relational trials. Error bars indicate SEM. * $p<0.001$.

\section{Results \\ Behavioral results}

\section{Item memory}

The mean proportions of remember and familiar responses to target words and first words for item-specific and relational trials are shown in Figure $2 A, B$. For both first words and target words, overall hit rates (remember plus familiar responses), as well as rates of remember responses, were significantly higher than the corresponding false-alarm rates [overall hits: first words $\left(t_{(12)}=\right.$ $2.57 ; p<0.05)$, target words $\left(t_{(12)}=2.27 ; p<0.05\right)$; remember responses: first words $\left(t_{(12)}=9.53 ; p<0.001\right)$, target words $\left.\left(t_{(12)}=7.98 ; p<0.001\right)\right]$. Our next analyses focused on memory for the target words, because this is where the two trial types differed with respect to item-specific and relational processing. Despite these differences, subsequent memory for target words was nearly identical across the two tasks (remember responses, $t_{(12)}<1$; familiar responses, $\left.t_{(12)}<1\right)$. These data were also used to estimate the contribution of recollection and familiarity to recognition, under an independence assumption (Yonelinas and Jacoby, 1995). As with the raw responses, estimates of recollection (item-specific mean, 0.43; relational mean, 0.41 ) and familiarity (item-specific mean, 0.35 ; relational mean, 0.36 ) for target words did not differ across trial types (both trial types, $t_{(12)}<1$ ). The close match in recognition performance across the two trial types suggests that both tasks engaged a similar depth of processing (Craik and Lockhart, 1972) of the target word.

A key difference between the two encoding tasks was that the relational task required participants to explicitly relate the target word to the first word, whereas the item-specific task solely involved processing of the target word. We therefore predicted that recognition memory for first words should be better on relational trials than on item-specific trials. As shown in Figure $2 B$, the rate of remember responses was significantly higher on relational tri- 
Table 1. Mean proportion of responses made on the associative recognition test for item-specific and relational trials

\begin{tabular}{llllll}
\hline & Correct & & & Incorrect & \\
\cline { 2 - 3 } & High confidence & Low confidence & & High confidence & Low confidence \\
\hline Item specific & 0.08 & 0.52 & & 0.04 & 0.36 \\
Relational & 0.35 & 0.38 & & 0.03 & 0.23 \\
\hline
\end{tabular}

Note that due to a small number of trials with no response, the proportions for the relational trials do not add to one

als, compared with item-specific trials $\left(t_{(12)}=5.15 ; p<0.001\right)$. No significant differences were observed in the rate of familiar responses $\left(t_{(12)}=1.03 ; p>0.05\right)$. Results were similar for estimates of recollection and familiarity; recollection of the first word was higher on relational trials than on item-specific trials (item-specific mean, 0.41 ; relational mean, $0.54 ; t_{(12)}=5.15 ; p<$ 0.001 ), but familiarity did not significantly differ across trial types (item-specific mean, 0.35 ; relational mean, $0.40 ; t_{(12)}=1.31 ; p>$ 0.05).

\section{Associative memory}

The results from the associative recognition test are shown in Table 1. We predicted that building a relationship between the first word and target word in relational trials should result in better memory for the word pairs than processing the target word in isolation in the item-specific trials. Consistent with our prediction, the percentage of correct responses (both high and low confidence) for relational trials was significantly greater than for item-specific trials (relational trials, $73 \%$; item-specific trials, $\left.59 \% ; t_{(13)}=3.89 ; p<0.005\right)$. In both tasks, the percentage of correct responses was significantly greater than the $50 \%$ expected by chance (relational trials: $t_{(13)}=6.78, p<0.001$; item-specific trials: $\left.t_{(13)}=4.21, p<0.005\right)$. To eliminate guesses from our fMRI analysis, we focused on high-confidence responses. For both relational and item-specific trials, the percentage of correct high-confidence responses was significantly greater than the percentage of incorrect high-confidence responses (relational trials: $t_{(13)}=8.15, p<0.001$; item-specific trials: $t_{(13)}=3.02, p<$ 0.005). Furthermore, the percentage of correct high-confidence responses was significantly higher for pairs encoded on relational trials than for pairs encoded on item-specific trials $\left(t_{(13)}=3.88\right.$; $p<0.005$ ).

Collectively, these behavioral results demonstrate that although item-specific and relational processing had an equivalent effect on memory for the target word, memory for the association between the first word and the target word was significantly increased on relational trials compared with item-specific trials. Because our fMRI subsequent memory analyses examined both item memory for the target words and associative memory for first word-target word pairs, we examined the overlap between these measures. Of the pairs given high-confidence responses on the associative recognition test, $55 \%$ of the target words also received a remember response on the item test; of the target words given a remember response on the item test, $31 \%$ were also part of a pair that was correctly recognized with high confidence. Thus, although there was some overlap between the two types of memory, there were a large number of trials for which the target word was remembered but the association was not, and vice versa.

\section{fMRI results}

Based on the idea that the DLPFC contributes to LTM formation by active encoding of relationships between items, our first prediction was that DLPFC activation should be increased during relational encoding trials compared with item-specific trials. In

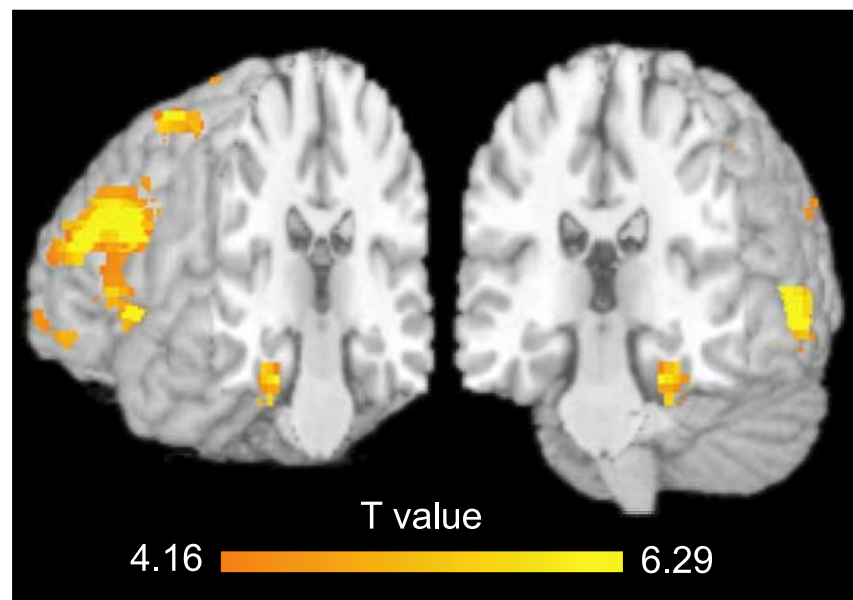

Figure 3. Regions showing greater target word activation on relational than on itemspecific trials. These include the bilateral DLPFC (BA 46) and VLPFC (BA 44, 45 and 47) as well as the left PHC and fusiform gyrus.

all of our analyses, we examined activation during processing of the target word, because this is the point at which subjects performed an encoding task that was either relational or item specific. Consistent with our prediction, this contrast revealed extensive activation in the lateral PFC (Fig. 3), including the ventrolateral prefrontal regions along the inferior frontal gyrus (BA 44, 45, and 47) (Amunts et al., 1999) and dorsolateral prefrontal regions along the middle frontal gyrus (at or near BA 46) (Rajkowska and Goldman-Rakic, 1995). This analysis also revealed activation in other regions that have been consistently implicated in successful item memory encoding, including the left PHC and left fusiform gyrus (for a complete list of regions identified in this contrast, see Table 2).

The results described above are consistent with the idea that the DLPFC is disproportionately engaged during active encoding of relationships between items. As noted earlier, psychological studies indicate that processing such relationships can promote the encoding of associations between items in LTM (Bower, 1970). Accordingly, our next set of analyses tested the prediction that DLPFC activity during encoding of the target word would correlate with subsequent LTM for the association between target word and the first word. We used the results from the contrast between relational and item-specific trials described above to define an ROI in the left DLPFC (BA 46) and examined activity in the ROI as a function of subsequent associative memory performance.

To identify whether activation in this region was correlated with subsequent associative memory performance, we contrasted target word activation on trials for which the pair of words was recognized with high confidence against activation on trials for which the pair elicited either a low-confidence response or an incorrect high-confidence response on the associative memory test. The trial-averaged time course for the DLPFC ROI is shown in the first panel of Figure $4 \mathrm{~A}$. Consistent with our prediction, DLPFC activation during processing of the target word was higher on trials for which the association was remembered with high confidence $\left(t_{(12)}=4.13 ; p \leq 0.005\right)$. One potential concern with this contrast is that of the pairs remembered with high confidence, more came from relational trials than item-specific trials. To ensure that the subsequent memory effect was not secondary to between-task differences, we examined this same contrast for target words, but restricted the contrast to relational trials only. 
Table 2. Local maxima of regions active at the target word during relational trials relative to item-specific trials

\begin{tabular}{|c|c|c|c|c|c|}
\hline$t$ value & $x$ & $y$ & $z$ & Location & $\mathrm{BA}$ \\
\hline 10.47 & -49 & 10 & 24 & Inferior frontal gyrus & 44 \\
\hline 9.76 & -10 & 14 & 56 & Pre-supplementary motor area & 6 \\
\hline 8.74 & -52 & 35 & 18 & Inferior/middle frontal gyrus & $45 / 46$ \\
\hline 7.64 & -56 & -56 & 0 & Middle temporal gyrus & $21 / 37$ \\
\hline 7.4 & -32 & 24 & 0 & Inferior frontal gyrus/anterior insula & $47 / 12$ \\
\hline 7.35 & -28 & -28 & -24 & Parahippocampal gyrus & $35 / 36$ \\
\hline 7.25 & 46 & 14 & 24 & Inferior frontal gyrus & 44 \\
\hline 7.13 & 32 & 21 & -4 & Inferior frontal gyrus/anterior insula & $47 / 12$ \\
\hline 7.08 & -38 & -35 & -21 & Fusiform gyrus & 37 \\
\hline 7.07 & -14 & 10 & 7 & Caudate nucleus & \\
\hline 6.72 & -35 & -52 & 46 & Intraparietal sulcus & 40 \\
\hline 6.66 & -32 & 14 & 56 & Superior frontal gyrus & 6 \\
\hline 6.64 & -52 & 49 & -7 & Lateral orbital gyrus & 47 \\
\hline 6.44 & 49 & 38 & 32 & Middle frontal gyrus & 9 \\
\hline 6.06 & -38 & 4 & 56 & Superior frontal sulcus & 8 \\
\hline 5.19 & -38 & -42 & 42 & Intraparietal sulcus & 40 \\
\hline 5.1 & -14 & 7 & 66 & Superior frontal gyrus & 6 \\
\hline 5 & -14 & 28 & 28 & Dorsal anterior cingulate gyrus & 32 \\
\hline 4.95 & -7 & 10 & 28 & Dorsal anterior cingulate gyrus & 32 \\
\hline 4.8 & -46 & -56 & -10 & Middle temporal gyrus & 37 \\
\hline 4.49 & -49 & 7 & 56 & Precentral sulcus & 8 \\
\hline
\end{tabular}

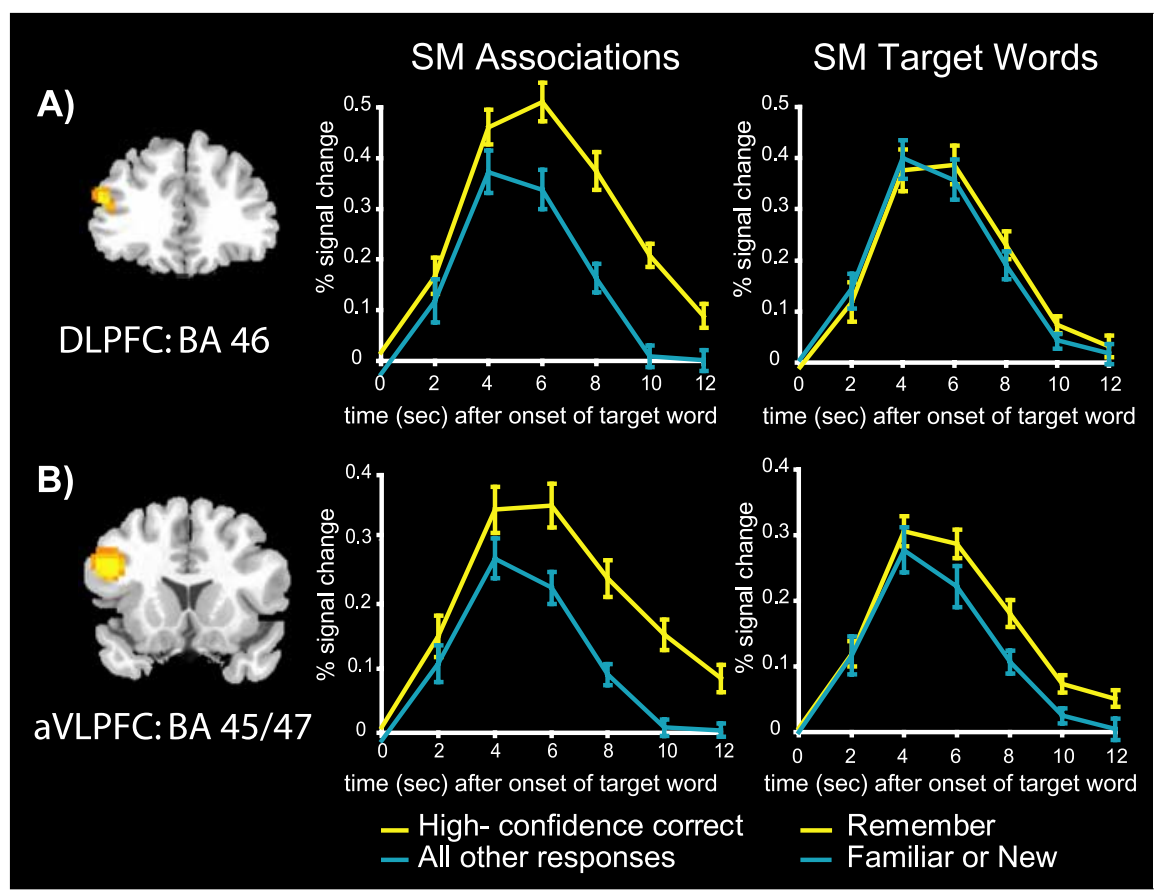

Figure 4. Time courses of activation in prefrontal ROls during processing of the target word as a function of subsequent memory (SM) for associations and items. Left, Time courses are separately averaged for trials in which the first word, target word association was remembered with high confidence and trials in which an incorrect or a low-confidence response was given. Right, Time courses are separately averaged for trials in which the target word was subsequently recollected and for trials in which the target word was subsequently recognized as familiar or forgotten. $\boldsymbol{A}$, Activation in the left DLPFC (BA 46) ROI was correlated with subsequent LTM for associations but not significantly correlated with subsequent item memory. $\boldsymbol{B}$, Activation in the left aVLPFC (BA 45/47) ROI was correlated with subsequent LTM for items and associations. Results in the pVLPFC (BA 44) were similar to those shown for the aVLPFC. Error bars indicate SEM.

This contrast essentially tested the prediction that, during relational encoding, the magnitude of DLPFC recruitment should predict subsequent associative memory performance. Consistent with this prediction, DLPFC activation was significantly higher on relational trials for which the association was remembered with high confidence $\left(t_{(12)}=2.22 ; p \leq 0.05\right)$ (for trial-averaged time courses, see supplemental material, available at www.jneurosci.org). We next examined participants' responses from the study phase on the relational task to ensure that the effect was not driven by a failure to perform the task on trials in the low-confidence/incorrect associative memory bin. The trials with incorrect responses were approximately evenly split between the high-confidence correct bin and the incorrect/low-confidence bin (for statistical analysis, see supplemental material, available at www.jneurosci.org), supporting the idea that it is indeed a subsequent memory effect rather than a taskrelated effect.

Results from the above analysis are consistent with the idea that the DLPFC is involved in processes that contribute to LTM for associations between items, but they could also suggest a more general role in promoting successful memory formation. If so, we would expect that DLPFC activation would be enhanced during processing of target words that were subsequently remembered. We therefore contrasted activation elicited by target words that were subsequently recollected (i.e., associated with a remember response) against target words that were subsequently recognized on the basis of familiarity or forgotten (i.e., rated familiar or new). As shown in the second panel of Figure $4 A$, activation in the DLPFC ROI was not significantly correlated with subsequent memory for the target word $\left(t_{(12)}<\right.$ $1)$. To determine whether the DLPFC would show a subsequent memory effect for target words in a less stringent contrast, we compared activation for target words subsequently given a remember response against target words subsequently given a new response; again, activation in the DLPFC was not predictive of item memory $\left(t_{(11)}<1\right)$.

The results described above suggest that DLPFC activation was correlated with successful memory for associations but not with successful item memory. To test whether the associative subsequent memory effect was statistically larger than the item subsequent memory effect, we performed a memory success (successful, unsuccessful) by test-type (item recognition, associative recognition) ANOVA on target word activation; this analysis showed a significant interaction both when all trials were included $\left(F_{(1,11)}=23.34 ; p<0.005\right)$ as well as when only relational trials were included in the associative memory contrast $\left(F_{(1,11)}=4.99 ; p<0.05\right)$.

We next examined activation in other regions that were identified in the relational versus item-specific contrast, to determine whether these regions would exhibit similar patterns of activa- 
tion. ROIs were defined in four regions that have been consistently implicated in successful LTM encoding (Paller and Wagner, 2002; Ranganath and Blumenfeld, 2007): a relatively anterior area of the left VLPFC (aVLPFC; BA 45 and 47), a more posterior area of the left VLPFC (pVLPFC; BA 44), as well as the left PHC and the left fusiform gyrus. Target word activation in both VLPFC ROIs (aVLPFC: $t_{(12)}=5.75, p<0.001$; pVLPFC: $t_{(12)}=$ 5.53, $p<0.001)$ and the PHC $\left(t_{(12)}=2.47 ; p<0.05\right)$ was increased on trials for which the association was subsequently recognized with high confidence compared with trials that elicited incorrect or low-confidence responses. This same pattern was evident in the VLPFC ROIs (aVLPFC: $t_{(12)}=3.89, p<0.005$; pVLPFC: $\left.t_{(12)}=2.30, p<0.05\right)$ when this comparison was restricted to pairs encoded on relational trials; the PHC showed a similar trend $\left(t_{(12)}=2.09 ; p=0.06\right)$. Unlike the DLPFC, activation in the aVLPFC and PHC was also increased for target words that later elicited remember responses compared with words that elicited familiar or new responses (aVLPFC: $t_{(12)}=2.30, p<$ 0.05; PHC: $\left.t_{(12)}=2.63, p<0.05\right)$. A similar trend was evident in the $\mathrm{pVLPFC}$ ROI but did not reach significance $\left(t_{(12)}=1.93 ; p=\right.$ $0.08)$. Finally, activation in the fusiform gyrus ROI differed from the prefrontal and PHC ROIs in that it did not show differential activation related to either subsequent associative memory $\left(t_{(12)}=1.67 ; p>0.05\right)$, or subsequent item memory $\left(t_{(12)}<1\right)$.

Although the hippocampus was not identified as showing differential activation between relational and item-specific trials, a large number of previous studies have implicated the hippocampus in relational memory encoding (Davachi et al., 2003; Ranganath et al., 2004; Prince et al., 2005; Eichenbaum et al., 2007). We therefore ran exploratory analyses on an anatomically defined ROI in the left hippocampus to investigate whether activation in this region was modulated by the encoding task or by encoding success. Analyses of parameter estimates and examination of trial-averaged time courses revealed that target word activation in this ROI did not significantly differentiate between encoding tasks or as a function of performance on the item memory test. Additionally, parameter estimates indexing target word activation did not differ as a function of subsequent associative memory, but a late-emerging memory effect was apparent on close inspection of the time courses (for details, see supplemental material, available at www.jneurosci.org).

\section{Discussion}

This experiment tested the hypothesis that the DLPFC promotes successful LTM formation by actively building associations between items during memory encoding. We compared activation between a relational encoding task that involved assessment of the relationship between two unrelated items and an itemspecific encoding task that involved assessment of the semantic attributes of an item in isolation. These two tasks had dissociable effects on item and associative memory such that memory for the target items encoded in both tasks was equivalent, but memory for the association between the items encoded on each trial was significantly higher in the relational condition. Critically, DLPFC activity was disproportionately increased during the relational encoding task compared with the item-specific task, and its activation during processing of the target word was predictive of subsequent associative memory but not item memory. The VLPFC and PHC were also preferentially activated during the relational task relative to the item-specific task, but activation in these areas was predictive of both subsequent associative memory and item memory, indicating a more general role for these regions in LTM formation.

\section{Item-specific versus relational encoding}

Research on the cognitive neuroscience of memory encoding has built on the levels of processing framework (Craik and Lockhart, 1972), which proposed that "elaborative" or "deep" processing of an item (e.g., semantic processing) strongly facilitates later memory, whereas minimal or "shallow" processing of an item (e.g., processing the perceptual attributes of an item) impacts memory to a lesser extent. Subsequent psychological theories have emphasized the match between what is encoded and the conditions and cues presented at retrieval [for encoding specificity principle, see Tulving and Thomson (1973); for transfer appropriate processing, see Morris et al., (1977)]. Consistent with this idea, Bower (1970) found that relational and item-specific encoding elicited equivalent levels of item memory but that relational encoding resulted in superior memory for associations between the words. This indicates that item-specific and relational encoding processes contribute to LTM formation in different ways, with relational encoding disproportionately promoting memory for associations between items. Results from the present study were fully consistent with this idea; whereas recognition of the target word (based on recollection and/or familiarity) was equivalent across the two tasks, associative recognition performance was significantly higher on relational trials. Consequently, differences in brain activity between the two tasks cannot be attributed simply to a difference in depth of processing. If this were the case, one would expect the relational condition to elicit increased recollection of the target word as well as its associate.

\section{DLPFC activity and relational memory encoding}

The current study was unique in examining relational processing during LTM encoding in that we manipulated both the way in which items were encoded (item-specific vs relational) as well as the way in which they were tested (item vs associative recognition). These manipulations revealed two important findings: DLPFC activation was (1) increased during relational compared with item-specific encoding and (2) predictive of subsequent memory for the associations between words, but not for the words themselves. Collectively, these findings are consistent with the idea that the DLPFC may contribute to LTM formation by building relationships between items.

Our findings are generally consistent with results from studies that have investigated neural correlates of memory encoding. For example, other studies of memory encoding have reported DLPFC activation during interactive imagery of pairs of words (Jackson and Schacter, 2004), during intentional encoding of pairs of images (Achim and Lepage, 2005), during reordering of word triplets based on a semantic rule (Davachi et al., 2001; Wagner et al., 2001b; Blumenfeld and Ranganath, 2006), and while subjects judged the number of semantic associations present among a list of three words (Addis and McAndrews, 2006). All of these findings share a common basic principle: DLPFC activation was obtained in tasks that involved active processing of relationships between items that were active in working memory.

Few studies have reported significant subsequent memory effects in the DLPFC, but our findings may explain why this is the case. Under encoding or retrieval conditions that emphasize memory for distinctive features of items and are insensitive to memory for inter-item associations, DLPFC activity may not be correlated with successful LTM formation. Our results demonstrate that the DLPFC will exhibit subsequent memory effects on tests that are sensitive to relational processing performed at encoding. Other evidence consistent with this idea has come from previous studies that observed DLPFC subsequent memory ef- 
fects after relational but not item-based working memory processing (Blumenfeld and Ranganath, 2006) and studies showing that DLPFC encoding activation was correlated with subsequent performance on tests sensitive to memory for inter-item associations (Staresina and Davachi, 2006; Summerfield et al., 2006).

One alternative explanation of the present results is that the DLPFC might be engaged under any circumstance that leads to subsequent recollection. Another possibility is that DLPFC activation supports LTM formation more specifically through its role in refreshing previously active representations (Raye et al., 2002; Johnson et al., 2004). However, these hypotheses would lead to the prediction that DLPFC activation should also be correlated with subsequent item recognition performance. This prediction is inconsistent with the finding that DLPFC activation (unlike activation in the VLPFC and PHC) was more specifically linked with subsequent memory for associations [for converging results, see Blumenfeld and Ranganath (2006)].

\section{VLPFC and memory encoding}

VLPFC activation has been reported in numerous studies of LTM encoding [for a listing, see Blumenfeld and Ranganath (2007)], and consistent with these studies, we obtained activation in an anterior area of the VLPFC (BA 45/47) that was predictive of subsequent memory for both items and associations, with a similar pattern evident in a more posterior area (BA 44) of the VLPFC. Although the pattern of subsequent memory effects in these VLPFC regions was somewhat different from those observed in the DLPFC (where activity was related to subsequent memory for associations, but not for items), our statistical analysis did not reveal evidence for a qualitative difference between the two regions (supplemental material, available at www. jneurosci.org). Thus, our results, along with those of other recent studies of memory encoding (Blumenfeld and Ranganath, 2006; Staresina and Davachi, 2006), suggest that it may be appropriate to consider a hierarchical model to explain prefrontal recruitment during memory encoding. More specifically, regions in the VLPFC may be recruited during any encoding operation that places emphasis on processing of distinctive item features or resolution of competition among items (Thompson-Schill et al., 1997; Wagner et al., 2001a). During relational encoding, DLPFC networks may be additionally recruited to activate or inhibit representations of relationships between items that are currently being processed through VLPFC operations (Ranganath and Blumenfeld, 2007).

\section{PHC}

A number of studies have reported that encoding activity in the $\mathrm{PHC}$ is predictive of subsequent recollection-based recognition but not subsequent familiarity-based recognition (Eichenbaum et al., 2007). Based on these findings, and evidence suggesting a role for the PHC in the processing of spatial (Bohbot et al., 1998; Janzen and van Turennout, 2004) and nonspatial (Bar and Aminoff, 2003) context, Eichenbaum et al. (2007) suggested that the PHC may encode context representations that can subsequently support the experience of recollection. The subsequent memory results obtained in the current study are consistent with this prediction: activation in the PHC was increased during encoding that led to subsequent remember responses on the item test as well as during encoding that led to subsequent recollection of correct word pairs. The PHC also showed overall increases in activation during relational trials relative to item-specific trials. It is possible that the imagery involved in performing the relational task (i.e., deciding whether one item could fit inside the other) resulted in the development of distinctive context representations, compared with those produced in the item-specific task (animacy judgments). This might explain increased recruitment of PHC on relational trials, in light of previous findings demonstrating increased $\mathrm{PHC}$ activation during processing of items associated with unique contexts (Bar and Aminoff, 2003; Aminoff et al., 2006).

\section{Conclusion}

In conclusion, our findings support the hypothesis that the DLPFC is critical for active processing of relationships between items, which in turn may promote LTM for associations. Thus, a common mechanism can potentially explain the recruitment of DLPFC activation in working memory (Postle et al., 1999; Wagner et al., 2001b; Barde and Thompson-Schill, 2002; Bor et al., 2004) and LTM (Blumenfeld and Ranganath, 2006; Staresina and Davachi, 2006; Summerfield et al., 2006) tasks. We speculate that many laboratory studies of LTM encoding have not tied DLPFC activity to successful LTM formation, because these studies typically minimize relational processing at encoding and deemphasize the role of inter-item associations at retrieval. Our results demonstrate that the DLPFC likely plays an important role in LTM outside of the laboratory, because real-life episodic memories are richly detailed and will benefit from relational processing among different aspects of an event as they unfold over time.

\section{References}

Achim AM, Lepage M (2005) Neural correlates of memory for items and for associations: an event-related functional magnetic resonance imaging study. J Cogn Neurosci 17:652-667.

Addis DR, McAndrews MP (2006) Prefrontal and hippocampal contributions to the generation and binding of semantic associations during successful encoding. NeuroImage 33:1194-1206.

Aminoff E, Gronau N, Bar M (2007) The parahippocampal cortex mediates spatial and nonspatial associations. Cereb Cortex, in press.

Amunts K, Schleicher A, Burgel U, Mohlberg H, Uylings HB, Zilles K (1999) Broca's region revisited: cytoarchitecture and intersubject variability. J Comp Neurol 412:319-341.

Bar M, Aminoff E (2003) Cortical analysis of visual context. Neuron 38:347-358.

Barde LH, Thompson-Schill SL (2002) Models of functional organization of the lateral prefrontal cortex in verbal working memory: evidence in favor of the process model. J Cogn Neurosci 14:1054-1063.

Blumenfeld RS, Ranganath C (2006) Dorsolateral prefrontal cortex promotes long-term memory formation through its role in working memory organization. J Neurosci 26:916-925.

Blumenfeld RS, Ranganath C (2007) Prefrontal cortex and long-term memory encoding: an integrative review of findings from neuropsychology and neuroimaging. Neuroscientist, in press.

Bohbot VD, Kalina M, Stepankova K, Spackova N, Petrides M, Nadel L (1998) Spatial memory deficits in patients with lesions to the right hippocampus and to the right parahippocampal cortex. Neuropsychologia 36:1217-1238.

Bor D, Cumming N, Scott CE, Owen AM (2004) Prefrontal cortical involvement in verbal encoding strategies. Eur J Neurosci 19:3365-3370.

Bower G (1970) Imagery as a relational organizer in associative learning. J Verb Learn Verb Behav 9:529-533.

Boynton GM, Engel SA, Glover GH, Heeger DJ (1996) Linear systems analysis of functional magnetic resonance imaging in human V1. J Neurosci 16:4207-4221.

Cocosco C, Kollokian V, Kwan R, Evans (1997) A brainweb: online interface to a 3D MRI simulated brain database. NeuroImage 5:s425.

Craik FIM, Lockhart RS (1972) Levels of processing: a framework for memory research. J Verb Learn Verb Behav 12:599-607.

Daselaar SM, Prince SE, Cabeza R (2004) When less means more: deactivations during encoding that predict subsequent memory. NeuroImage 23:921-927.

Davachi L, Maril A, Wagner AD (2001) When keeping in mind supports 
later bringing to mind: neural markers of phonological rehearsal predict subsequent remembering. J Cogn Neurosci 13:1059-1070.

Davachi L, Mitchell JP, Wagner AD (2003) Multiple routes to memory: distinct medial temporal lobe processes build item and source memories. Proc Natl Acad Sci USA 100:2157-2162.

Desjardins AE, Kiehl KA, Liddle PF (2001) Removal of confounding effects of global signal in functional MRI analyses. NeuroImage 13:751-758.

Eichenbaum H, Yonelinas AP, Ranganath C (2007) The medial temporal lobe and recognition memory. Annu Rev Neurosci, in press.

Genovese CR, Lazar NA, Nichols T (2002) Thresholding of statistical maps in functional neuroimaging using the false discovery rate. NeuroImage 15:870-878.

Handwerker DA, Ollinger JM, D’Esposito M (2004) Variation of BOLD hemodynamic responses across subjects and brain regions and their effects on statistical analyses. NeuroImage 21:1639-1651.

Jackson III O, Schacter DL (2004) Encoding activity in anterior medial temporal lobe supports subsequent associative recognition. NeuroImage 21:456-462.

Janzen G, van Turennout M (2004) Selective neural representation of objects relevant for navigation. Nat Neurosci 7:673-677.

Johnson MK, Mitchell KJ, Raye CL, Greene EJ (2004) An age-related deficit in prefrontal cortical function associated with refreshing information. Psychol Sci 15:127-132.

Morris CD, Bransford JD, Franks JJ (1977) Levels of processing versus transfer appropriate processing. J Verb Learn Verb Behav 16:519-533.

Otten LJ, Rugg MD (2001) When more means less: neural activity related to unsuccessful memory encoding. Curr Biol 11:1528-1530.

Otten LJ, Henson RN, Rugg MD (2002) State-related and item-related neural correlates of successful memory encoding. Nat Neurosci 5:1339-1344.

Paller KA, Wagner AD (2002) Observing the transformation of experience into memory. Trends Cogn Sci 6:93-102.

Postle BR, Berger JS, D’Esposito M (1999) Functional neuroanatomical double dissociation of mnemonic and executive control processes contributing to working memory performance. Proc Natl Acad Sci USA 96:12959-12964.

Prince SE, Daselaar SM, Cabeza R (2005) Neural correlates of relational memory: successful encoding and retrieval of semantic and perceptual associations. J Neurosci 25:1203-1210.

Rajkowska G, Goldman-Rakic PS (1995) Cytoarchitectonic definition of prefrontal areas in the normal human cortex: II. Variability in locations of areas 9 and 46 and relationship to the Talairach coordinate system. Cereb Cortex 5:323-337.

Ranganath C, Blumenfeld RS (2007) Prefrontal cortex and human memory: an integrated account of results from neuropsychological and neuroimaging studies of working- and long-term memory. In: Learning and memory: a comprehensive reference (Eichenbaum H, ed). Oxford, UK: Elsevier.

Ranganath C, Cohen MX, Dam C, D'Esposito M (2004) Inferior temporal, prefrontal, and hippocampal contributions to visual working memory maintenance and associative memory retrieval. J Neurosci 24:3917-3925.

Raye CL, Johnson MK, Mitchell KJ, Reeder JA, Greene EJ (2002) Neuroimaging a single thought: dorsolateral PFC activity associated with refreshing just-activated information. NeuroImage 15:447-453.

Rorden C, Brett M (2000) Stereotaxic display of brain lesions. Behav Neurol 12:191-200.

Staresina BP, Davachi L (2006) Differential encoding mechanisms for subsequent associative recognition and free recall. J Neurosci 26:9162-9172.

Summerfield C, Greene M, Wager T, Egner T, Hirsch J, Mangels J (2006) Neocortical connectivity during episodic memory formation. PLoS Biol 4:e128.

Thompson-Schill SL, D’Esposito M, Aguirre GK, Farah MJ (1997) Role of left inferior prefrontal cortex in retrieval of semantic knowledge: a reevaluation. Proc Natl Acad Sci USA 94:14792-14797.

Tulving E (1962) Subjective organization in free recall of "unrelated" words. Psychol Rev 69:344-354.

Tulving E (1985) Memory and consciousness. Can Psychol 26:1-12.

Tulving E, Thomson DM (1973) Encoding specificity and retrieval processes in episodic memory. Psychol Rev 80:352-373.

Wagner AD, Davachi L (2001) Cognitive neuroscience: forgetting of things past. Curr Biol 11:R964-967.

Wagner AD, Pare-Blagoev EJ, Clark J, Poldrack RA (2001a) Recovering meaning: left prefrontal cortex guides controlled semantic retrieval. Neuron 31:329-338.

Wagner AD, Maril A, Bjork RA, Schacter DL (2001b) Prefrontal contributions to executive control: fMRI evidence for functional distinctions within lateral prefrontal cortex. NeuroImage 14:1337-1347.

Worsley KJ, Friston KJ (1995) Analysis of fMRI time-series revisited-again. NeuroImage 2:173-181.

Yonelinas AP, Jacoby LL (1995) Dissociating automatic and controlled processes in a memory-search task: beyond implicit memory. Psychol Res 57:156-165. 